\title{
El derecho a la libertad religiosa en los sistemas regionales de protección de derechos humanos de Europa y América ${ }^{1}$
}

\section{The Right to Freedom of Religion in the Regional Human Rights Protection Systems in Europe and America}

\author{
Ruth Martinón Quintero² \\ Universidad de La Laguna (España) \\ ORCID: https://orcid.org/0000-0001-8013-7215
}

Recibido: 12-01-2020

Aceptado: 07-06-2020

\section{Resumen}

El derecho a la libertad religiosa ha sido abordado con diferente intensidad por el Tribunal Europeo de Derecho Humanos y por las instituciones protectoras del Sistema Interamericano, la Comisión y la Corte. Sin embargo, las diferencias entre ambos ámbitos de protección de los derechos humanos no son solo cuantitativas porque responden a realidades históricas, políticas y sociales distintas. Aun así, también se dan puntos de encuentro en los sujetos titulares y obligados, en la perfilación de su contenido y en sus límites. Lo más llamativo del Tribunal europeo es el recurso constante al margen de apreciación nacional ante la diversidad de posturas de los Estados miembros del Consejo de Europa; y, en el Sistema Interamericano, la vinculación del derecho a la protección de los más vulnerables, bien a través de la cosmovisión de los pueblos indígenas, bien por su relación con el trabajo de muchos activistas de la sociedad civil con los más pobres.

\footnotetext{
${ }^{1}$ El trabajo se enmarca en el proyecto de investigación "Teorías de la justicia y derecho global de los derechos humanos" (referencia PID2019-107172RB-I00), financiado por el Ministerio de Ciencia e Innovación de España.

2 (ruth.martinon@ull.es) Profesora de la Universidad de La Laguna y Directora Académica de su Centro de Documentación Europea. Es doctora por la Universidad Carlos III de Madrid, licenciada en Derecho y en Ciencia Política. Entre sus publicaciones más recientes destaca "La legitimidad normativa de la Corte Interamericana de Derechos Humanos como tribunal internacional”, Andamios, Vol. 17, 42 (2020), pp. 121-145.
} 
Palabras-clave: derecho a la libertad religiosa, Tribunal Europeo de Derechos Humanos, Sistema Interamericano de Derechos Humanos.

\begin{abstract}
The right to religious freedom has been approached with different intensity by the European Court of Human Rights and by the protective institutions of the Inter-American System, the Commission and the Court. However, the differences between the two protective spheres of human rights are not only quantitative, because they respond to different historical, political and social realities. Even so, there are also meeting points in the titular and obligated subjects, in the outline of their content and in their limits. The most striking aspect of the European Court is the constant recourse to the margin of national appreciation in the face of the diversity of positions of the member states of the Council of Europe; and, in the Inter-American System, the linking of the right to protection of the most vulnerable, either through the worldview of indigenous peoples, or because of its relationship with the work of many civil society activists with the poorest.
\end{abstract}

Key-words: right to freedom of religion, European Court of Human Rights, American System of Human Rights.

El derecho de la intolerancia es, por tanto, absurdo y bárbaro; es el derecho de los tigres, y es mucho más horrible, porque los tigres solo desgarran para comer, y nosotros nos hemos exterminado por unos párrafos.

Voltaire, Tratado sobre la tolerancia, 1763.

\title{
1. Introducción
}

El objetivo de este trabajo es mostrar los puntos coincidentes en la perfilación pretoriana del derecho a la libertad religiosa (LR) en los sistemas internacionales de protección de derechos humanos europeo y americano como muestra del diálogo posible entre ambos. Al tiempo se ponen de manifiesto las inevitables diferencias fruto de realidades históricas, sociales, culturales, políticas y jurídicas distintas.

En el caso del Consejo de Europa, el trabajo se centra en la jurisprudencia del Tribunal Europeo de Derechos Humanos (TEDH) porque es profuso (lo que, de hecho, no nos permite un repaso exhaustivo, sino representativo), aunque con frecuencia los casos se reconducen a otros derechos como, por ejemplo, el derecho a la vida privada y familiar, o el derecho de asociación. 
En el caso del Sistema Interamericano de Derechos Humanos (SIDH) vamos a tener en cuenta tanto los pronunciamientos de la Comisión Interamericana de Derechos Humanos (CIDH) como los de la Corte Interamericana de Derechos Humanos (Corte IDH), tanto contenciosos como consultivos, por el reducido número de casos en este ámbito.

Existen algunas explicaciones sobre esta diferencia cuantitativa entre ambos sistemas. Los órganos del SIDH han centrado su trabajo en la violación de los derechos esenciales de la vida y de la libertad de las personas, así como en la tutela de los derechos políticos, que fueron lesionados de manera grave y masiva por las dictaduras militares que predominaron durante décadas en la región ${ }^{3}$. Asimismo, tradicionalmente la intolerancia religiosa no ha sido un problema en América, salvo en casos puntuales ${ }^{4}$. En cualquier caso, ese conocimiento limitado que se ha tenido del derecho a la LR de manera autónoma y principal no resta gravedad a las violaciones sufridas.

La abundancia de jurisprudencia del TEDH sobre este derecho desde 1993, en el cual se produjo la primera y famosa sentencia del caso Kokkinakis ${ }^{5}$, parece evidenciar la significativa presencia de lo religioso en las sociedades europeas. Hay tensiones abundantes en los países del Este recientemente incorporados al Consejo de Europa, pero también en los países occidentales, donde ha proliferado la diversidad religiosa debido a la inmigración, a veces en contraste con el uniformismo de antaño ${ }^{6}$. Así, una de las razones de la litigiosidad parece estar en la dificultad de abrir vías para la convivencia de confesiones religiosas con mucho peso histórico en un Estado con otras confesiones que se abren paso en la actualidad ${ }^{7}$, y la necesidad de que el Estado garantice esa convivencia entre las diversas religiones en pro de una sociedad democrática ${ }^{8}$.

\footnotetext{
${ }^{3}$ Héctor Fix-Zamundio, "La libertad religiosa en el Sistema Interamericano de protección de los derechos humanos", [En VV. AA.: La libertad religiosa. Memoria del IX Congreso internacional de Derecho canónico, México D. F., Instituto de Investigaciones Jurídicas de la Universidad Autónoma de México, 1996, pp. 499-510, p. 131]; Luis Francisco Cervantes G., "Los principios generales sobre la libertad religiosa en la jurisprudencia de los sistemas europeo, interamericano y costarricense de protección de los derechos humanos", Senderos: revista de ciencias religiosas y pastorales, 93, (2009), pp. 271-309, p. 131.

${ }^{4}$ Fernando Arlettaz, "La libertad religiosa en el Sistema Interamericano de derechos humanos", Revista Internacional de Derechos Humanos, 1 (2011), pp. 39-58, p. 43.

5 TEDH, Caso Kokkinakis contra Grecia, S. 25 mayo 1993.

6 Ángel G. Chueca Sancho, "El derecho humano a la libertad de religión y convicciones en una Europa intercultural”, [En Teoría de la Justicia y Derechos Fundamentales. Estudios en Homenaje al profesor Gregorio Peces-Barba. Volumen III, Madrid, Dykinson, 2008, pp. 297-318], p. 299.

${ }^{7}$ Lorenzo Martín-Retortillo Baquer, "La libertad religiosa en la reciente jurisprudencia del Tribunal Europeo de Derechos Humanos", Anuario de Derecho Eclesiástico del Estado, XXVI (2010), pp. 287-332, p. 289.

${ }^{8}$ Chueca Sancho, op. cit., p. 217.
}

Araucaria. Revista Iberoamericana de Filosofia, Política, Humanidades y Relaciones Internacionales, año $23, \mathrm{n}^{\circ} 46$. Primer cuatrimestre de 2021. Pp. 591-612. ISSN 1575-6823 e-ISSN 2340-2199 https://dx.doi.org/10.12795/araucaria.2021.i46.29 
En todo caso, la globalización parece ligada al protagonismo del fenómeno religioso, no solo por el incremento de la pluralidad de las sociedades estatales generado por las migraciones, sino por las oportunidades tecnológicas y psicosociales que la globalización proporciona a los movimientos religiosos ${ }^{9}$.

\section{Regulación del derecho a la LR en el Consejo de Europa y en el SIDH}

El Convenio para la protección de los Derechos y de las Libertades Fundamentales de 4 de noviembre de 1950 (CEDH) recoge en su artículo 9 el derecho a la libertad de pensamiento, de conciencia y de religión.

El artículo 14 del mismo Tratado asegura el goce de los derechos y libertades reconocidos en el mismo sin distinción alguna, incluyendo por razones de religión. Lo cual se reitera en la prohibición general de discriminación del artículo primero del Protocolo $12^{10}$, es decir, no solo respecto a los derechos y libertades del CEDH, sino en relación con el goce de todo derecho previsto en la Ley.

Cuando el Protocolo Primero del CEDH introdujo el derecho a la instrucción en su artículo 2, lo hizo añadiendo la obligación del Estado de respetar el derecho de los padres a asegurar esta educación y esta enseñanza conforme a sus convicciones religiosas ${ }^{11}$.

En el SIDH el derecho a la LR se recoge expresamente en el artículo 2 de la Declaración Americana de los Derechos y Deberes del Hombre; y, de manera mucho más amplia, en el artículo 12 de la Convención Americana de Derechos Humanos de 22 de noviembre de 1969 (CADH). El artículo 1.1 de dicho instrumento recoge la prohibición de discriminación en razón de credo y de religión.

\section{Sujetos del derecho a la libertad religiosa}

En sus orígenes, el derecho a la LR es estrictamente individual, de modo que los grupos religiosos no tienen más potestades que las "que sus miembros han acordado otorgarles por medio del contrato de asociación que les ha dado origen" y "los tratados internacionales de derechos humanos hoy existentes son tributarios de este modo de concebir la LR" $"$. Tanto el artículo 12 de la CADH como el artículo 9 del CEDH comienzan con la expresión "Toda persona".

\footnotetext{
9 Rafael Palomino, "Libertad religiosa y libertad de expresión”, Ius Canonicum, XLIX, 98 (2009), pp. 509-548, p. 511.

${ }^{10}$ Protocolo $\mathrm{n}^{\circ} 12$ al CEDH (Roma, 4 nov. 2000).

11 Protocolo Adicional al CEDH (París, 20 marzo 1952).

12 Fernando Arlettaz, "El individuo como titular de la libertad religiosa: aproximación a un problema de filosofía jurídica”, [En Ma Carmen Barranco Avilés, Óscar Celador Angón, Félix Vacas Fernández, coords.: Perspectivas actuales de los sujetos de Derechos, Madrid, Dykinson y Universidad Carlos III de Madrid, 2012, p. 303-315], pp. 305 y 306.
}

Araucaria. Revista Iberoamericana de Filosofia, Politica, Humanidades y Relaciones Internacionales, año $23, \mathrm{n}^{\circ} 46$. Primer cuatrimestre de 2021. Pp. 591-612. ISSN 1575-6823 e-ISSN 2340-2199 https://dx.doi.org/10.12795/araucaria.2021.i46.29 
En el ámbito del Consejo de Europa, aunque el artículo 9 del CEDH no menciona las confesiones religiosas, se ha entendido que su LR puede considerarse garantizada de forma implícita en la mención de la libertad de manifestar la propia religión "colectivamente"13.

Al principio la Comisión de Derecho Humanos (Comisión) restringió el derecho a la LR a las personas físicas. En la actualidad se produce una equiparación en la titularidad del derecho de las personas físicas y las personas jurídicas de modo que una confesión religiosa puede ser considerada "víctima" a los efectos del $\mathrm{CEDH}^{14}$. Esto es clave en la configuración de la autonomía de las comunidades religiosas como elemento indispensable para el pluralismo en una sociedad democrática ${ }^{15}$, elemento fundamental de la concepción del TEDH en este ámbito.

En el SIDH, la expresión “Toda persona” parece ceñir el titular del derecho a la LR a las personas físicas, puesto que no puede ser interpretada de otro modo de acuerdo con el artículo 1.2 de la CADH que define la persona como todo ser humano.

Sin embargo, la dimensión colectiva del derecho se ha puesto de manifiesto desde varias perspectivas como, por ejemplo, en el reconocimiento del derecho a la personalidad jurídica de sus grupos. El tener en cuenta la realidad del grupo puede basarse en la necesidad de proteger a los grupos más vulnerables y a las minorías culturales, cuyos derechos no están garantizados por la simple declaración de derechos individuales en abstracto ${ }^{16}$.

De hecho, recientemente, la Corte IDH confirmó en una opinión consultiva ${ }^{17}$ que las personas jurídicas no tenían derechos ante el SIDH, excepto los sindicatos y los pueblos indígenas en ciertos casos específicos. En el caso de estos últimos, su identidad y ciertos derechos individuales, como el derecho a la propiedad o a su territorio, solo pueden ejercerse a través de la comunidad a la cual pertenecen.

Consideramos que es importante detenerse en cómo, en el ámbito del SIDH, el derecho a la LR en relación con los pueblos indígenas ha servido para completar la protección de estas comunidades. Ello se ha llevado cabo, esencialmente, a través de la vinculación de su religiosidad a sus territorios, y la protección de estos por la Corte IDH a través de una modulación del derecho a la propiedad del artículo 21 de la $\mathrm{CADH}$, de modo que no solo se protege la

${ }^{13}$ Isidoro Martín Sánchez, "Las confesiones religiosas y su autonomía en el Tribunal Europeo de Derechos Humanos", Encuentros Multidisplinares, 26 (2014), pp. 1-9, p. 1.

${ }^{14}$ La consideración de víctima de una violación es fundamental para poder presentar una demanda ante el TEDH de acuerdo con el artículo 34 del CEDH. TEDH, Caso Delegación de Moscú del Ejército de Salvación c. Rusia, S. 5 oct. 2006, aptos. 63-70.

${ }^{15}$ TEDH, Caso Fernández Martínez c. España, S. 12 jun. 2014, apdo. 127.

${ }^{16}$ Arlettaz, 2012, op. cit., pp. 308-312.

${ }^{17}$ Corte IDH. Titularidad de derechos de las personas juridicas en el Sistema Interamericano de Derechos Humanos. OC-22/16, 26 feb. 2016, párs. 83 y 106.

Araucaria. Revista Iberoamericana de Filosofí, Política, Humanidades y Relaciones Internacionales, año $23, \mathrm{n}^{\circ} 46$. Primer cuatrimestre de 2021. Pp. 591-612. ISSN 1575-6823 e-ISSN 2340-2199 https://dx.doi.org/10.12795/araucaria.2021.i46.29 
propiedad en su sentido clásico, sino también formas de propiedad y posesión colectivas.

\begin{abstract}
Las tierras tradicionales y los recursos naturales no sólo son el principal medio de subsistencia de estos grupos, sino que además constituyen un elemento integrante de su cosmovisión, religiosidad y, por ende, de su identidad cultural. $[\ldots]$

La protección de las tierras tradicionales ha sido realizada a partir del derecho de propiedad, y no de la LR, aunque las creencias religiosas de los pueblos indígenas hayan sido mencionadas en los fundamentos de las sentencias. [...] También la CIDH ha señalado la relación entre cultura, espiritualidad y posesión de las tierras tradicionales. Ha dicho que esto es así porque en las tierras tradicionales se encuentran cementerios ancestrales, lugares de significado religioso y sitios ceremoniales. Por ello, las limitaciones a la propiedad indígena también pueden afectar el derecho al ejercicio de la propia religión y, en consecuencia, los Estados tienen la obligación de garantizar a los pueblos indígenas la conservación de sus propias formas de religiosidad, lo que incluye la expresión pública de las creencias y el acceso a los sitios sagrados ${ }^{18}$.
\end{abstract}

En el Caso Masacres de Río Negro se condenó al Estado por violación, entre otros, de la integridad personal en relación con el derecho a la LR por el daño causado por no poder realizar sus rituales fúnebres tanto por la desaparición de los cuerpos de los fallecidos, como por la inundación de sus tierras de enterramiento, consideradas sagradas ${ }^{19}$.

En el Caso Masacre Plan de Sánchez, la Corte IDH condenó al Estado por la violación, entre otros, del derecho de la LR, por el impedimento y consiguiente daño que sufrieron los supervivientes de la matanza para enterrar a sus muertos de acuerdo con sus ritos religiosos. De modo que, entre las reparaciones, se ordenó al Estado pagar "el mantenimiento y mejoras en la infraestructura de la capilla en la cual las víctimas rinden tributo a las personas ejecutadas en la Masacre Plan de Sánchez"20.

También en otros casos, aunque no se llega a considerar que el derecho objeto de protección hayan sido las creencias religiosas, se trata de aspectos que se tienen en cuenta al establecer las reparaciones por considerar que ha causado un especial sufrimiento a las víctimas. $\mathrm{O}$, simplemente, se reconoce la afectación a la vida religiosa de las víctimas de violaciones de derechos humanos, pero sin llegar a un pronunciamiento autónomo sobre el artículo 12 de la $\mathrm{CADH}^{21}$.

\footnotetext{
${ }_{18}$ Arlettaz, 2011, op. cit., p. 53-54.

19 Corte IDH, Caso Masacres de Río Negro vs. Guatemala. Excepción Preliminar, Fondo, Reparaciones y Costas, S. 4 sept. 2012, párs. 153 a 165, y punto resolutivo 4.

${ }^{20}$ Corte IDH. Caso Masacre Plan de Sánchez Vs. Guatemala. Reparaciones. S. 19 sept. 2004, pár. 104.

${ }^{21}$ Corte IDH, Caso miembros de la aldea Chichupac y comunidades vecinas del municipio de Rabinal vs. Guatemala, S. 30 nov. 2016, párs. 194 y 197.
} 
Respecto al sujeto pasivo del derecho a la LR, en el ámbito del Consejo de Europa es el Estado, con obligaciones tanto negativas (compromiso de abstenerse de cualquier injerencia en los derechos garantizados por el CEDH) como positivas de proteger el derecho de religión ${ }^{22}$ incluso frente a ataques provenientes de particulares. Así, se condenó a Georgia por su inacción frente a la violencia sufrida por los testigos de Jehová durante varios años ${ }^{23}$. Si bien, en esto, como en otras cuestiones que veremos posteriormente, es importante la repercusión de la doctrina del margen de apreciación nacional (MAN) ${ }^{24}$.

El Derecho del SIDH solo se ocupa de la exigencia de responsabilidad a los Estados, pero estos deben garantizar también el cumplimiento del derecho de LR por parte de los particulares. Los Estados son responsables por la violación directa del derecho, pero también si pudiendo garantizar su libre y pleno ejercicio como es su obligación (artículo 1.1 CADH), no lo hacen. Sirve de ejemplo el caso en el que, ante acciones de hostigamiento contra la comunidad judía venezolana, la CIDH entendió que el Estado no había hecho lo suficiente al respecto y le conminó a adoptar las medidas necesarias a fin de garantizar el pleno respeto de la LR en su territorio y a continuar investigando los incidentes mencionados ${ }^{25}$.

\section{Contenido del derecho a la libertad religiosa en ambos sistemas}

\subsection{El Consejo de Europa}

Los numerosos casos que han llegado al TEDH en materia de LR le han permitido abordarla ampliamente y asentar unos principios básicos que delimitan el bien jurídico protegido y las posibles limitaciones del derecho. El primer párrafo del artículo 9 refiere en primer lugar el contenido del derecho ad intra, que es el ámbito más protegido y el cual no permite restricciones, a diferencia de las manifestaciones, tanto individuales como colectivas, que sí pueden ser limitadas de acuerdo con el segundo párrafo del mismo artículo.

El artículo 9.1 del CEDH establece el derecho a la libertad de pensamiento, de conciencia y de religión, abarcando en posición de igualdad ${ }^{26}$ todo tipo de

${ }^{22}$ Ian Leigh, "New trends in Religious Liberty and the European Court of Human Rights", en Ecclesiastical Law Society, 12 (2020), pp. 266-279, p. 267; Agustín García Ureta, "Libertad de pensamiento, de conciencia y de religión", [En Iñaki Lasagabaster Herrarte, dir.: Convenio Europeo de Derechos Humanos. Comentario sistemático, Pamplona, Thomson Reuters, 2015, p. 439-509], p. 444-445.

$23 \mathrm{TEDH}$, Caso de 97 miembros de la Congregación de Testigos de Jehová de Gldani contra Georgia, S. 3 mayo 2007, apdos. 133-135.

24 TEDH, Caso Palomo Sánchez y otros c. España, S. 12 sept. 2011, apdos. 58 y 59. TEDH, Caso Eweida y otros c. Reino Unido, S. 15 ene. 2013, apdo. 84.

${ }^{25}$ CIDH, Informe Anual 2008, 25 feb. 2009, Cap. IV. Venezuela, párs. 364-367.

${ }^{26}$ Agustín García Ureta, "Libertad de pensamiento, de conciencia y de religión”, [En Lasagabaster Herrarte, Iñaki: Convenio Europeo de Derechos Humanos. Comentario sistemático. Pamplona:

Araucaria. Revista Iberoamericana de Filosofía, Política, Humanidades y Relaciones Internacionales, año 23, $\mathrm{n}^{\circ} 46$. Primer cuatrimestre de 2021. Pp. 591-612. ISSN 1575-6823 e-ISSN 2340-2199 https://dx.doi.org/10.12795/araucaria.2021.i46.29 
convicciones personales consistentes (que son más que meras opiniones) ${ }^{27}$, "modos de entender cómo se ha de conducir una persona en su vida individual o social y la actuación de acuerdo con ellas" 28 , sin que se haya visto la necesidad jurisprudencial de dar una definición al respecto.

En una de sus citas más famosas, el TEDH dijo que la libertad de pensamiento, de conciencia y de religión constituye una de las bases de una sociedad democrática en el sentido del CEDH. En su dimensión religiosa figura entre los elementos más esenciales de la identidad de los creyentes y de su concepción de la vida, pero es también un bien precioso para los ateos, los agnósticos, los escépticos o los indiferentes. Es una manifestación del pluralismo, claramente conquistado en el curso de siglos, consubstancial a nuestra sociedad ${ }^{29}$.

Por tanto, los principios que han de regir este derecho parten de la necesidad de garantizar la pluralidad como valor máximo de una sociedad democrática, a través de la neutralidad e imparcialidad del Estado ${ }^{30}$, el cual ha de operar como garante de la tolerancia mutua y la autonomía de las confesiones religiosas.

Aunque se trata de un ámbito en el que la amplia diversidad de posturas entre los Estados miembros del Consejo de Europa ha llevado a un amplio MAN en las limitaciones a las manifestaciones, estos principios básicos estarán en todo el control ejercido por el TEDH.

En lo concerniente al ámbito interno, se recoge el derecho absoluto e incondicional a profesar y a cambiar de religión (o a no profesar ninguna), sin que el Estado pueda inmiscuirse nunca en ello. Esta expresión de la LR supone que el Estado no puede adoctrinar en ninguna creencia religiosa ${ }^{31}$ (principio de neutralidad), ni forzar a manifestar ${ }^{32}$ o a que quede de manifiesto ${ }^{33}$ ninguna opción religiosa; ni valorar la legitimidad de las creencias religiosas o las formas de expresión de esas creencias ${ }^{34}$.

Respecto a la objeción de conciencia (especialmente referida al servicio militar obligatorio), el TEDH ha modificado la postura de la Comisión que no la reconocía como parte del derecho a la LR del artículo 9 del CEDH (sino como una opción por la que pueden decantarse libremente los Estados parte) sobre la base de que el CEDH es un instrumento vivo que debe atender a la

\footnotetext{
Thomson Reuters, 2015. Tercera edición, p. 439-509], p. 445.

27 Agustín García Ureta, "Libertad de pensamiento, de conciencia y de religión", [En Iñaki Lasagabaster Herrarte, dir.: Convenio Europeo de Derechos Humanos. Comentario sistemático, Pamplona, Thomson Reuters, 2015, p. 439-509], p. 445.

${ }_{28}$ María José Roca, "Impacto de la jurisprudencia del TEDH y la Corte IDH sobre libertad religiosa", Revista Española de Derecho Constitucional, 110 (2017), pp. 253-281, p. 257.

29 TEDH, Caso Kokkinakis contra Grecia, S. 19 abril 1993, apdo. 31.

${ }^{30}$ Aunque no se prohíben las religiones de Estado, sí que se prohíben las acciones que conculcan dicha neutralidad (García Ureta, op. cit., pp. 455-456).

31 TEDH, Caso Kjeldsen, Busk Madsen y Pedersen contra Dinamarca, S. 7 dic. 1975, apdo. 53.

32 TEDH, Caso Sinan Işik contra Turquía, S. 2 feb. 2010, apdos. 42-43.

33 TEDH, Caso Alexandridis contra Grecia, S. 21 feb. 2008, apdos. 35 y 38.

${ }^{34}$ Por todas, TEDH, Caso Eweida y otros contra el Reino Unido, S. 15 ene. 2013, apdo. 81.
} 
realidad de la mayoría de los Estados parte en cada momento, admitiendo así la oposición al servicio militar cuando exista un conflicto insalvable con las convicciones auténticas y profundas ${ }^{35}$.

En cuanto al proselitismo, el TEDH ha aceptado que el derecho a la LR incluye las acciones para convencer "al vecino"36 siempre que no se haga en prevalencia de la posición (por ejemplo de militares respecto a sus subordinados $)^{37}$, con presiones inaceptables o haciendo uso del acoso o la violencia. Por tanto, el TEDH admite el proselitismo entre iguales, pero no cuando se realiza a través de presiones ilícitas a través de lavado de cerebro o aprovechamiento de autoridad ${ }^{38}$.

En la interacción de la LR con la libertad de expresión reconocida en el artículo 10 del $\mathrm{CEDH}$, el TEDH ha tenido que llevar a cabo la ponderación correspondiente con un principio inequívoco: la libertad de expresión no abarca las expresiones que incitan al odio ${ }^{39}$.

En las primeras sentencias aceptó un amplio MAN respecto a las restricciones estatales establecidas para proteger los sentimientos religiosos de los creyentes admitiendo la prohibición de películas satíricas ${ }^{40} \mathrm{u}$ otro tipo de ataques genéricos a los creyentes de una religión, o la sanción de actos de desprecio a personajes relevantes de determinada confesión ${ }^{41}$.

Sin embargo, la jurisprudencia del TEDH ha evolucionado hacia la reducción del MAN que admitía las restricciones a la libertad de expresión que ofendían sentimientos religiosos (aun cuando no hubiera incitación al odio o a la violencia) ${ }^{42}$ a favor de la tutela de la libertad de expresión y la necesidad de que los grupos religiosos toleren la negación de sus creencias e incluso la propagación de otras doctrinas hostiles a su fe siempre que no se inste al odio o la intolerancia religiosos ${ }^{43}$.

El TEDH ha jugado un papel importante en la configuración de los delitos de odio (basados, entre otros, en prejuicios religiosos) como límite al derecho

35 TEDH, Caso Bayatyan contra Armenia, S. 27 oct. 2009, apdos. 108 y 110.

36 TEDH, Caso Kokkinakis contra Grecia, S. 25 mayo 1993.

37 TEDH, Caso Larissis y otros contra Grecia, S. 24 de feb. 1998.

${ }^{38}$ Gloria Fernández Arribas, "La Libertad religiosa en la Jurisprudencia el Tribunal Europeo de Derechos Humanos", [En Abraham Barrero Ortega y Manuel José Terol Becerra, coords.: La libertad religiosa en el Estado Social, Valencia, Tirant lo Blanch, 2009, pp. 443-462], p. 452.

39 TEDH, Caso E. S. contra Austria, S. 25 oct. 2018, apdo. 43.

40 Tanto en el Caso Otto-Preminger Institut contra Austria (TEDH, S. 20 dic. 1994) como en el Caso Wingrove contra Reino Unido (TEDH, S. 25 nov. 1996) se trató de sátiras o burlas al cristianismo que resultaron ofensivas.

${ }^{41}$ Así, por ejemplo, en el Caso E. S. contra Austria (TEDH, S. 25 oct. 2018), el TEDH consideró que las reflexiones sobre las tendencias pedófilas de Mahoma en una conferencia no estaban amparadas por el artículo 10 del CEDH porque éste no amparaba afirmaciones en hechos inciertos (apdos. 51-55).

${ }^{42}$ Zoila Combalía, "Los conflictos entre libertad de expresión y religión: tratamiento jurídico del discurso del odio", Anuario de Derecho Eclesiástico del Estado, XXXI (2015), pp. 355-379, pp. 362-365.

${ }^{43}$ Por todos: TEDH, Caso Tagiyev y Huseynov contra Azerbaijan, S. 5 dic. 2019, apdo. 44. 
a la libertad de expresión ${ }^{44}$. Esta evolución se ha explicado porque lo contrario "acabaría por difuminar el contenido de la libertad de expresión en beneficio de las confesiones dominantes y de los intereses políticos"45.

Uno de los aspectos más controvertidos radica en los símbolos religiosos, principalmente por su confrontación con el principio de laicidad de Francia, Suiza y Turquía, pero también en otros Estados.

En general, el TEDH ha permitido las limitaciones estatales en virtud del MAN y en detrimento de tests rigurosos de proporcionalidad, hasta el punto de que ha generado soluciones contradictorias. Por ello se le ha criticado el vaciamiento del derecho y la falla en el cumplimiento en su misión de garantizar el respeto estatal ${ }^{46}$.

En el caso del pañuelo islámico, ha considerado que en las sociedades democráticas el Estado puede limitar su uso y ha abordado este problema especialmente en el ámbito educativo de universidades ${ }^{47}$ y escuelas ${ }^{48}$. También se ha permitido imponer el criterio escolar en confrontación con el criterio de los padres en pro de la integración social y la evitación de la exclusión de los alumnos extranjeros ${ }^{49}$.

Sin embargo, la máxima expresión del MAN en relación con el laicismo se encuentre en la Sentencia del Caso S.A.S., que acabó considerando como no violación el artículo 9 del CEDH la prohibición de cubrirse el rostro en lugares públicos de la Ley 11 de octubre de 2010 de Francia, pese a los argumentos críticos desarrollados por el propio TEDH al respecto ${ }^{50}$. Unos años antes, sin embargo, había considerado desproporcionado e innecesario la prohibición turca de uso de vestimenta religiosa en lugares públicos ${ }^{51}$.

El asunto Lautsi también puede considerarse paradigmático de los problemas del TEDH con los símbolos religiosos, en particular, con la presencia del crucifijo en las aulas. En un primer momento, una sala del TEDH concluyó que se trataba de una infracción del artículo 2 del Protocolo y del artículo 9 del $\mathrm{CEDH}^{52}$. Posteriormente, la Gran Sala consideró que realmente no podía

44 Elena Avilés Hernández, "El discurso del odio como límite a la libertad de expresión: Análisis de la jurisprudencia del TEDH y su influencia en el Tribunal constitucional español", [En Carmen Sánchez Hernández y otros, dirs.: La influencia de la jurisprudencia del TEDH en el derecho interno, Valencia, Tirant lo Blanch, 2019, pp. 55-82], pp. 65 y ss.

${ }^{45}$ Martín Sánchez, op. cit., in fine.

46 Sergio Alejandro Fernández Parra, "El margen nacional de apreciación y el contenido de la libertad de pensamiento, conciencia y religión en el Convenio Europeo de Derechos Humano", Eunomía, 17 (2019-2020), pp. 68-99, pp. 70 y 93.

${ }^{47}$ TEDH, Caso Leyla Sahin contra Turquía, S. 10 nov. 2005, en la que se permitió la prohibición del uso del velo en la Universidad de Estambul.

${ }^{48}$ TEDH, Casos Dogru y Kervanci contra Francia, Ss. 4 dic. 2008, en las que se autorizó la prohibición del velo en clase de educación física.

49 TEDH, Caso Osmanoğlu y Kocabaş contra Suiza, S. 10 ene. 2017, apdo. 96.

50 TEDH, Caso S.A.S. contra Francia, S. 1 jul. 2014, apdos. 145-157.

51 TEDH, Caso Ahmet Arslan y otros contra Turquía, S. 23 feb. 2010, apdo. 49.

52 TEDH, Caso Lautsi y otros contra Italia, S. 3 nov. 2009. 
confirmar que el crucifijo tuviese alguna influencia en los alumnos y, que ante el escenario de los Estados parte, debía primar también aquí el MAN ${ }^{53}$. Sin embargo, había inadmitido el caso de una profesora que reclamaba el derecho a portar el velo en Suiza porque podía afectar la conciencia de sus estudiantes ${ }^{54}$.

En los conflictos vinculados al puesto de trabajo, en general no se reconoce el derecho a abandonar el trabajo por una fiesta religiosa ${ }^{55} \mathrm{o}$ el derecho al ajuste de los días laborales para poder disfrutar o cumplir con la misma ${ }^{56}$, y se admite la posibilidad de que se le pida a un trabajador algún tipo de evidencia de su creencia para poder ampararse a excepciones laborales concretas, en una excepción del derecho a no manifestar la opción religiosa ${ }^{57}$.

El problema en este ámbito se puede resumir en hasta qué punto el Estado puede imponer una política razonable de acomodación de las diferentes creencias religiosas en el puesto de trabajo ${ }^{58}$.

En el Caso Eweida, el TEDH consideró que sí había habido violación de los artículo 9 y 13 del CEDH respecto a la prohibición de una trabajadora de llevar una pequeña cruz, porque primó el derecho a manifestar sus propias convicciones religiosas frente a la imagen corporativa de la empresa; al tiempo que consideró que no hubo violación en la prohibición del mismo objeto en una enfermera por razón de seguridad médica; ni tampoco cuando no se permitió a otros trabajadores dejar de realizar los trabajos contrarios a sus creencias como registrar matrimonios homosexuales o prestar asesoramiento sexual a parejas del mismo sexo ${ }^{59}$.

En el Caso Ebrahimian ${ }^{60}$ tampoco se consideró al Estado responsable por la no renovación del contrato a la demandante por no querer quitarse el pañuelo en el lugar de trabajo.

Todo ello pone de manifiesto la necesidad de la ponderación en cada caso concreto, aunque la solución está clara en casos extremos de persecución religiosa por parte del Estado ${ }^{61}$. Lo cierto es que en estos conflictos subyace la dicotomía entre la mera tolerancia de las diferentes confesiones que no permite las excepciones a la norma general, y el respeto de las diferentes manifestaciones

\footnotetext{
${ }^{53}$ TEDH, Caso Lautsi y otros contra Italia, S. 18 mar. 2011, apdos. 66 y 68-70.

54 TEDH, Caso Dahlab contra Suiza. Demanda 42393/98, S. 15 feb. 2001.

55 TEDH, Caso Kosteski contra Antigua República Yugoslava de Macedonia, S. 13 abril 2006, apdos. 39 y 46.

56 TEDH, Caso Franceso Sessa contra Italia, S. 3 abril 2012, en el que el Tribunal aceptó que las autoridades judiciales italianas no cambiaran la fecha de una vista que coincidía con una fiesta religiosa del abogado.

57 TEDH, Caso Kosteski contra Antigua República Yugoslava de Macedonia, S. 13 abril 2006, apdo. 45 .

${ }_{58}$ European Court of Human Rights, op. cit., p. 22.

59 TEDH, Caso Eweida y otros contra el Reino Unido, S. 15 ene. 2013, apdos. 94-95, 99-101, 106, 109.

60 TEDH, Caso Ebrahimian contra Francia, S. 26 nov. 2015.

${ }^{61}$ TEDH, Caso Ivanova contra Bulgaria, S. 12 abril 2007, apdos. 84-85.
} 
religiosas que sí podría suponer modelaciones de dichas normas generales, en una postura más generosa con las minorías.

Es en la manifestación colectiva del derecho a la LR donde se expresa de manera más evidente la autonomía de las confesiones religiosas y las correlativas neutralidad e imparcialidad del Estado respecto a las mismas, garantizando el pluralismo imprescindible de una sociedad democrática. Por ello, esta autonomía se encuentra en el núcleo de la concepción del TEDH de la LR y sin ella "todos los demás aspectos de la LR del individuo se encontrarían debilitados" $"$.

Aparece aquí el derecho a la personalidad jurídica. Son múltiples las sentencias de condena a los Estados que han impedido u obstaculizado el registro $\mathrm{y} / \mathrm{o}$ el otorgamiento de personalidad jurídica a grupos religiosos; frecuentemente, grupos minoritarios en el país del que se trate, por lo que suele vincularse también a un problema de discriminación ${ }^{63}$.

Según el TEDH los artículos 9 y 11 (derecho de asociación) del CEDH no garantizan a las iglesias determinado estatus legal, pero los Estados no pueden impedir u obstaculizar los procedimientos de Derecho interno necesarios para que las iglesias y sus miembros puedan manifestar libremente sus creencias ${ }^{64}$.

El Estado, en su deber de neutralidad e imparcialidad, no tiene ningún poder de apreciación sobre la legitimidad de las creencias religiosas ${ }^{65}$, de modo que su negativa a dicho reconocimiento ha de basarse en razones muy importantes y graves ${ }^{66}$. El Estado, como organizador neutral e imparcial del ejercicio de las diversas religiones, debe asegurar la tolerancia mutua entre los grupos opuestos, evitando todo tipo de violencia entre ellos ${ }^{67}$ pero sin suprimir las causas de las tensiones pues ello es consustancial al pluralismo que hay que proteger ${ }^{68}$.

La autonomía interna de las confesiones también supone que los Estados no pueden inmiscuirse en sus problemas internos para garantizar su unidad o decantándose por uno de los liderazgos parte de un conflicto ${ }^{69}$. Abarca la total libertad de las confesiones religiosas para determinar quiénes son sus miembros, sin que el artículo 9 del CEDH abarque el derecho de estos a la disidencia: en caso de desacuerdo doctrinal $\mathrm{u}$ organizacional entre una comunidad religiosa y sus miembros, la LR se expresa en el derecho de estos a abandonar libremente dicha comunidad ${ }^{70}$.

${ }^{62}$ TEDH, Caso Ebrahimian contra Francia, S. 26 nov. de 2015, apdo. 118.

${ }^{63}$ TEDH, Caso Delegación de Moscú del Ejército de Salvación contra Rusia, S. 5 oct. 2006, apdo. 97.

${ }^{64}$ TEDH, Caso Mirolubovs y otros contra Letonia, S. 15 sept. 2009, apdo. 80. c.

65 TEDH, Caso Iglesia Metropolitana de Bessarabia y otros contra Moldavia, S. 13 dic. 2001, apdo. 117.

${ }^{66}$ TEDH, Caso Iglesia moscovita de la cienciología, S. 5 abril 2007, apdo. 75

67 TEDH, Caso de 97 miembros de la Congregación de Testigos de Jehová de Gldani c. Georgia, S. 2 mayo 2007, pp. 139-142.

68 TEDH, Caso Leyla Şahin contra Turquía, S. 10 nov. 2005, apdos. 107-108.

69 TEDH, Caso Mirolubovs y otros contra Letonia, S. 15 sept. 2009, apdo. 80. f.

70 TEDH, Caso Mirolıbovs y otros contra Letonia, S. 15 de sep. 2009, apdo. 80. d. 
En el nombramiento de ministros religiosos y otro tipo de personal, como los profesores de religión, el TEDH ha de compatibilizar la protección del derecho de los grupos religiosos a su autonomía con la adecuada tutela de derechos fundamentales de trabajadores cuya vinculación profesional con una confesión religiosa hace que voluntariamente asuman una serie de deberes de lealtad especiales ${ }^{71}$.

La relación con el principio de igualdad y no discriminación por razón de religión se vincula a la misma existencia del derecho y es difícil no encontrarlo, aunque sea subliminalmente, en todos los conflictos. En los casos concretos en los que esta relación es protagonista son imprescindibles juicios complejos de proporcionalidad.

\subsection{Sistema Interamericano de Derechos Humanos}

En el ámbito del SIDH, lo primero que destaca en el contenido del derecho a la LR es que el artículo 12 abarca tanto la LR como de conciencia, sin que la CIDH ni la Corte IDH hayan establecido una distinción clara entre ambas o hayan dado una definición de religión.

También podemos colegir que este derecho abarca tanto la libertad ad intra de profesar una religión (cuando se establece la libertad de conservar o cambiar de religión), como el derecho ad extra de manifestar y practicar esa religión, esto es: la libertad de profesar y divulgar la religión, tanto individualmente como colectivamente, tanto en público como en privado, lo que implícita abarca "el culto, la enseñanza, las prácticas y el cumplimiento de ritos"72.

Finalmente, la última sección del artículo 12 incluye la libertad de educación religiosa entendida como el derecho de los padres y tutores a que sus hijos o sus alumnos reciban educación moral y religiosa de acuerdo con sus propias convicciones, lo que ha se entendido como límite para los Estados americanos en la regulación de este derecho en tanto no pueden imponer una religión a los menores de edad ${ }^{73}$.

Este aspecto fue uno de los más controvertidos en la redacción de la $\mathrm{CADH}^{74}$ y marca la principal diferencia con el artículo 9 del CEDH, si bien éste

${ }^{71}$ María José Valero Estarellas, “Autonomía institucional de las confesiones religiosas y derecho al respeto de la vida privada y familiar en Estrasburgo: la S. la Gran Sala del TEDH Fernández Martínez c. España”, Revista General de Derecho Canónico y Derecho Eclesiástico del Estado, 36 (2014), pp. 1-21, p. 15. TEDH, Caso Fernández Martínez contra España, S. 12 jun. 2014, apdo. 131 respecto al deber especial de lealtad.

${ }^{72}$ Fiz-Zamundio, op. cit., p. 503.

${ }^{73}$ Xiomara Lorena Romero Pérez, "La libertad religiosa en el Sistema Interamericano de Protección de los Derechos Humanos (Análisis Comparativo con el ordenamiento jurídico colombiano)", Revista Derecho del Estado, 29 (2012), pp. 215-232, p. 223.

${ }^{74}$ Laurence Burgorgue-Larse, "Le système interaméricain des droits de l'homme face aux religions", [En Robert Uerpmann-Wittzack, Evelyne Lagrange y Stefan Oeter, Religion and International Law. 
se complementó con el artículo 2 del Primer Protocolo Adicional que integra el derecho de los padres a garantizar la educación de sus hijos de acuerdo con sus convicciones religiosas y filosóficas: lo cual ha sido muy importante en el abordaje primigenio de este derecho ${ }^{75}$.

La CIDH ha tratado la LR en diversos informes, tanto anuales como de país, además de en algunos casos, especialmente graves los años de dictaduras militares.

En 1979, la CIDH consideró que el gobierno de Argentina había infringido, entre otros, el derecho de LR y de culto al prohibir toda actividad de los Testigos de Jehová ${ }^{76}$. En el Informe Anual 1979-1980, la CIDH también denunció la persecución de este grupo religioso en Paraguay ${ }^{77}$, lo que motivó un llamamiento de la Asamblea de la Organización de Estados Americanos a los estados miembros para que no impidieran el ejercicio del derecho a la libertad de credo y de culto ${ }^{78}$. En este caso, la CIDH consideró que la personalidad jurídica (que el gobierno paraguayo había quitado al grupo Testigos de Jehová) era parte de la LR en la medida en que es necesaria para permitir la participación en la vida civil como asociación religiosa.

Respecto a Cuba, los informes de la CIDH de la década de los ochenta señalaban que la Constitución cubana de 1976 permitía intervenir la reglamentación de las actividades religiosas e impedía invocar la LR si ésta interfería con los ideales marcados por la revolución.

Cuba es el país sobre el que más se ha ocupado la CIDH en el ámbito de la LR, al considerar que ésta sufría restricciones fundamentales: en lo referido a los medios de comunicación de masas y la educación ${ }^{79}$, y la discriminación por motivos de religión para la promoción laboral, adquisición de determinados bienes, acceso a la universidad o becas ${ }^{80}$. Tras la modificación de la Constitución cubana en septiembre de 1992 y el reconocimiento de la libertad de conciencia y religión (modificación que se considera resultado o impacto de la actividad

Living Together, Leiden-Boston, Brill Nijhoff, 2018, pp. 157-174], p. 166.

75 Durante muchos años (desde el Caso Kjeldsen, Busk Madsen y Pedersen contra Dinamarca con S.1 TEDH del 7 de diciembre de 1976 al Caso Kokkinakis contra Grecia, S.1 TEDH del 25 de mayo de 1993) el punto de referencia en materia religiosa estuvo en la interpretación de esta manifestación a través de la enseñanza y el vínculo entre el respeto de las convicciones de los padres en la enseñanza de sus hijos y el pluralismo en la educación, antecedente del pluralismo que se constituirá en bandera de la interpretación del artículo 9 del CEDH.

${ }^{76}$ CIDH, Caso 2137 Testigos de Jehová (Argentina), Informe 02/79 (véase Informe sobre la situación de los derechos humanos en Argentina, 11 abril 1980, Cap. X. B).

77 CIDH, Informe Amual 1979-1980, 2 oct. 1980, Cap. V. B, sección 10.

78 Asamblea General de la OEA. Resolución 444 (IX-0/79), aprobada en la duodécima sesión plenaria, 31 oct. 1979. En Actas y Documentos. Vol. I. Washington, D.C.: Secretaría general de la OEA, p. 87

${ }^{79} \mathrm{CIDH}$, La situación de los derechos humanos en Cuba. Séptimo Informe, 4 oct. de 1983, Cap. VII, párs. 26, 32 y 42.

${ }^{80}$ CIDH, Informe Anual 1985-1986, 26 sept. 1986, Cap. IV. 
del SIDH) ${ }^{81}$, cesaron las menciones a Cuba respecto a la LR, y en el año 2000 la CIDH celebró que se hubiesen permitido celebraciones religiosas ${ }^{82}$.

Sin embargo, en el Informe anual de 2013, la misma institución se hizo eco de denuncias de las Damas de Blanco, y de otras organizaciones religiosas, de sufrir diversos actos de agresión y hostigamiento por la asistencia a misa por parte de las fuerzas de seguridad cubanas en diversos puntos del país. También se denunció la prohibición de las prácticas y tradiciones culturales y religiosas de la población afrocubana ${ }^{83}$.

La CIDH también mostró su preocupación por las limitaciones que la Iglesia católica ha sufrido en El Salvador ${ }^{84}$, Nicaragua ${ }^{85}$, Guatemala ${ }^{86}$ y Paraguay ${ }^{87}$. Pone en relación la LR con la promoción de las doctrinas sociales y políticas que estén vinculadas a la visión teológica que sustenta una comunidad religiosa. De modo que en estos casos la persecución por motivos políticos, el impedimento de actividades políticas o de asistencia social a los más necesitados, o la prohibición a los religiosos de realizar propaganda política de crítica al gobierno, constituyen, además de la violación de otros derechos humanos básicos, una afectación a la $\mathrm{LR}^{88}$.

Dentro de esta concepción de la LR, encontramos uno de los casos más conocidos de la CIDH: el de Diana Ortiz. Se trató de una religiosa de origen norteamericano que en 1989 fue secuestrada, torturada y violada por parte de agentes del Estado, de acuerdo con lo considerado probado por la CIDH, debido al compromiso de la religiosa con los más pobres y, en especial, con los pueblos indígenas. Por todo ello, la CIDH responsabilizó al Estado guatemalteco de la violación de varios derechos, entre ellos el de la LR en la medida en que

Es probable que los ataques contra la Hermana Ortiz hayan tenido como objetivo castigarla y truncar sus actividades religiosas como misionera de la Iglesia y su labor con los grupos indígenas [...]. Además, debido a la vigilancia, amenazas, secuestro, tortura y violación de que fue objeto, regresó a los Estados Unidos para escapar de sus secuestradores y la violencia contra ella y no ha podido regresar a Guatemala por temor. Como resultado, se la ha privado del derecho de ejercer su derecho a la libertad de conciencia y de religión desempeñándose como misionera extranjera de la Iglesia Católica en Guatemala ${ }^{89}$.

\footnotetext{
${ }^{81}$ ROCA, op. cit., p. 255.

${ }^{82}$ CIDH, Informe Anual 2000, 16 abril 2001, Cap. IV. Cuba, pár. 6.

${ }^{83}$ CIDH, Informe Anual 2013, 31 dic. 2013, Cap. IV. B. párs. 177, 204 y 227.

${ }^{84} \mathrm{CIDH}$, Informe sobre los derechos humanos en El Salvador, 17 nov. 1978, Cap. VIII, párs. 11 et 14-17; y CIDH, Informe Anual 1979-1980, 2 oct. 1980, Cap. V. D, sección 4.

${ }^{85} \mathrm{CIDH}$, Informe sobre los derechos humanos en Nicaragua, 17 nov. 1979, Cap. VI.

${ }^{86} \mathrm{CIDH}$, Informe sobre los derechos humanos en la República de Guatemala, 13 oct. 1981. Cap. VI; y CIDH, Informe sobre los derechos humanos en la República de Guatemala, 3 oct. 1983, Cap. VI.

${ }^{87}$ CIDH, Informe Anual 1983-1984, 28 sept. 1984, Cap. IV. Paraguay, pár. 9.

${ }^{88}$ Arlettaz, 2011, op. cit., p. 46-47

${ }^{89} \mathrm{CIDH}$, Informe $N^{\circ} 31 / 96$, Caso 10.526, Guatemala, 16 oct. 1996, pár. 119.
} 
Hay que tener en cuenta que en el SIDH el derecho a la LR no abarca la objeción de conciencia. En el caso Cristian Daniel Sahli Vera, la víctima denunció la violación de la libertad de conciencia por haberse visto obligado a realizar el servicio militar. La CIDH interpretó que se reconoce el derecho a la condición de objetor de conciencia solamente cuando lo recoge la legislación interna. Por tanto, el reconocimiento de la objeción de conciencia forma parte del MAN en el SIDH ${ }^{90}$.

El único caso elevado a la Corte en el que se alegó de manera principal la violación del derecho a la LR fue el caso Olmedo Bustos ${ }^{91}$. Esta sentencia tuvo un gran impacto porque supuso la modificación de la constitución chilena. En este caso se denunció al Estado chileno por prohibir la proyección de la película La última tentación de Cristo, una decisión fundada en el art.19 de la Constitución Política de Chile de 1980, que permitía la censura previa.

El Estado defendió que la actuación era pertinente por considerar que la obra atentaba contra los sentimientos religiosos de los cristianos. La CIDH alegó la violación del derecho a la LR argumentando que la censura de la película había impedido que la sociedad accediera a una información que habría podido llevar a algunos individuos a mantener, cambiar o modificar sus creencias religiosas, lo cual fue descartado por la Corte IDH como no probado. La Corte IDH consideró que el derecho a la LR es uno de los cimientos de la sociedad democrática pero que en este caso no existía prueba que acreditase su violación. Se trataba de una violación de la libertad de expresión que no podía restringirse para evitar afectar los sentimientos religiosos.

Por tanto, el derecho a la LR de la CADH no prohíbe los actos que afectan a los sentimientos religiosos de la población. Lo único que la ley puede prohibir son la apología del odio religioso que constituya una incitación a la violencia, así como otras acciones ilegales similares contra una persona o grupo de personas por motivo de religión ${ }^{92}$.

Precisamente, en el Informe anual de 2017, la CIDH mostró su preocupación por las posturas oficiales intolerantes de algunos Estados que desean "prohibir la circulación de personas entre Estados en función de su religión, entre diversas otras medidas que promueven un clima favorable a los ataques raciales y crímenes de odio [que] aumentaron en algunos países"93.

También en el SIDH se ha puesto de manifiesto la relación fundamental entre el derecho a la LR y el principio de no discriminación. Sin embargo, la discriminación a menudo ocurre al combinar diferentes categorías vulnerables,

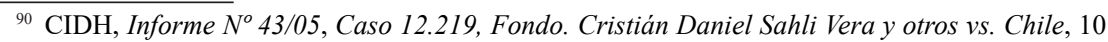
de marzo de 2005. CIDH, Informe Anual 2005, 27 feb. 2006, Cap. III. C.

${ }^{91}$ Corte IDH. Caso "La Última Tentación de Cristo”" (Olmedo Bustos y otros) vs. Chile. S. 5 feb. 2001, párs. 74-80.

${ }_{92}$ CIDH, Informe Anual 2013, 31 dic. 2013. Cap. IV, pár. 49.

${ }^{93}$ CIDH, Informe Anual 2017, 31 dic. 2017 Cap. IV. A, pár. 15. 
como los pueblos indígenas, los pobres y los defensores de estos grupos. En el Informe anual de 2014, la CIDH denunció la actuación policial (controles y trato posterior en la detención) con sesgo racista en Estados Unidos, considerando que ese sesgo incluye la discriminación por religión ${ }^{94}$.

\section{Límites del derecho a la libertad religiosa}

Tanto el artículo 9.2 del CEDH como el artículo 12.3 de la CADH recogen la posibilidad de limitar las manifestaciones externas de este derecho con redacciones muy similares.

El TEDH considera que el derecho de tener cualquier creencia religiosa y a cambiar de religión o creencia es absoluto e incondicional ${ }^{95}$. Sin embargo, puesto que la manifestación por una persona de su creencia religiosa puede tener un impacto en otras, existen los límites del artículo 9.2 que dispone que cualquier limitación de la manifestación de la religión o las creencias debe ser regulada por Ley y ser necesaria en una sociedad democrática en la consecución de uno o más objetivos. Es decir, puesto que en una sociedad democrática coexisten diversas religiones y convicciones personales, las restricciones son necesarias para conciliar los intereses de los distintos grupos y asegurar el respeto a las creencias de todos ${ }^{96}$.

El TEDH ha desarrollado un sistema, a veces criticado como demasiado mecánico ${ }^{97}$, con el que primero determina si ha habido injerencia estatal en el derecho y, si es así, si dicha injerencia cumple los requisitos del artículo 9.2 CEDH y, por tanto, es admisible.

De este modo, los límites al derecho se concretan en las injerencias que pueden realizar los Estados en el ejercicio de su deber de neutralidad como garantes del pluralismo, parte insoslayable de una sociedad democrática. El problema más relevante surge cuando esta prueba de proporcionalidad no se ha realiza con todo su rigor por el recurso al MAN que antepone consideraciones como el laicismo o las tradiciones religiosas mayoritarias de un Estado ${ }^{98}$.

Se considera que se cumple con el principio de legalidad si la norma que regula la limitación es accesible, precisa y previsible ${ }^{99}$, función que pueda cumplir también la jurisprudencia adecuada en materias no reguladas

${ }^{94}$ CIDH. Informe Anual 2014, 9 marzo 2015, Cap. IV, pár. 77.

95 TEDH, Caso Eweida y otros c. Reino Unido, S. 15 ene. 2013, apdo. 80.

${ }^{96}$ European Court of Human Rights. Research Division, op. cit., p. 7.

${ }^{97}$ Brett G. Scharffs, "Symposium Introduction: The Freedom of Religion and Belief Jurisprudence of the European Court of Human Rights: Legal, Moral, Political and Religious Perspectives", Journal of Law and Religion. Vol. 26, 1 (2010-2011), p. 258.

98 Fernández Parra, op. cit., pp. 90 y 93.

99 TEDH, Caso Leyla Şahin contra Turquía, S. 10 nov. de 2005, apdo. 91. 
suficientemente por Ley ${ }^{100}$. Se trata de una concepción sustancial, no formal, del término Ley ${ }^{101}$.

Además, la injerencia ha de justificarse mostrando que sirve para la seguridad pública, la protección del orden de la salud o de la moral públicas, o la protección de los derechos o libertades de los demás; y ha de ser necesaria en el marco de una sociedad democrática. Esto se concreta en que exista una necesidad social imperiosa que justifique la injerencia y que ésta sea proporcional al fin perseguido. Esta configuración ha permitido, por ejemplo, la prohibición del velo islámico ${ }^{102}$ o el turbante sij, o la cruz del cuello de una enfermera ${ }^{103}$; la prohibición de proselitismo cuando media relación de autoridad $^{104}$; etc.

En la CADH, respecto a las imitaciones del derecho a la LR debemos atender a varios aspectos. El párrafo 3 del artículo 12 de la CADH hace referencia, en primer lugar, a la necesidad de que toda limitación sea establecida por ley en sentido estricto y de manera precisa.

El "sentido estricto" significa entender como ley solamente "la ley adoptada por organismos elegidos democráticamente facultados por la Constitución, adheridos al bien común"105.

La "manera precisa" invalida cláusulas ambiguas como la de la antigua constitución cubana que declaró: "es ilegal y punible oponer la fe o la creencia religiosa a la revolución".

Como ha precisado la doctrina ${ }^{106}$, dicha limitación debe estar justificada en la necesidad en una sociedad democrática de "proteger la seguridad, el orden, la salud o la moral públicas o los derechos o libertades de los demás". Es una cláusula de orden público que trata de hacer compatible los intereses individuales protegidos por el derecho con el interés general de la sociedad y los intereses individuales de los otros ${ }^{107}$.

La Corte IDH se pronunció indirectamente sobre los límites de la LR en el caso Instituto de reeducación del menor, al afirmar que el derecho a la LR no puede limitarse por sufrir privación de libertad ${ }^{108}$. En dos opiniones consultivas ha precisado el alcance del artículo 27.2 de la $\mathrm{CADH}$, el cual incluye la libertad de conciencia y religión entre los derechos que no pueden ser suspendidos en estados de excepción (guerras, peligro público, emergencias...).

\footnotetext{
100 TEDH, Case of Kokkinakis v. Grecia, S. 25 mayo 1993, apdos. 40 y 41.

101 Fernández Arribas, op. cit., p. 455.

102 Por todas: TEDH, Caso Drogu contra Francia, S. 4 dic. 2008, apdo. 64.

103 TEDH, Caso Eweida y otros c. Reino Unido, S. 15 ene. 2013, apdos. 99-101.

104 TEDH, Caso Larissis y otros contra Grecia, S. 24 feb. 1998, apdo. 54.

105 Corte IDH. La expresión “Leyes” en el artículo 30 de la CADH. OC-6/86 9 mayo 1986, pár. 37.

106 Cervantes, op. cit., p. 132

107 Arlettaz, 2011, op. cit., p. 51.

108 Corte IDH. Caso “Instituto de Reeducación del Menor” vs. Paraguay. S. 2 sept. 2004, pár. 155.
} 
En la OC $8 / 87^{109}$ la Corte aclaró que los recursos judiciales del habeas corpus y amparo (artículos 7.6 y 25.1 Convención) constituyen garantías indispensables para proteger los derechos que no admiten suspensión durante los estados de excepción.

En la OC- $9 / 87^{110}$ precisó que, sin importar la denominación del recurso, no pueden suspenderse las garantías que protejan los derechos durante los estados de anormalidad. Esta prohibición de suspensión del derecho a la LR y las garantías destinadas a su protección durante los estados de anormalidad constituye una salvaguardia especial de $l^{a} L^{111}$ y una diferencia importante con el artículo 15 del CEDH.

\section{Reflexiones finales}

Aunque la CIDH y la Corte IDH no se han ocupado del derecho a la LR de manera prolija, sus pronunciamientos son suficientes para rastrear su caracterización principal y realizar cierta comparación con la labor del TEDH.

El artículo 9 del CEDH (junto al artículo 2 del Protocolo primero) y el artículo 12 de la CADH no son idénticos. Sin embargo, ambos han permitido una determinación equivalente de los elementos básicos del derecho.

Respecto a los titulares del derecho, la admisión del ius standi activo de las personas jurídicas es más amplia en el ámbito europeo. El SIDH también ha admitido el derecho a la personalidad jurídica de las confesiones religiosas y la titularidad colectiva de ciertos grupos, en especial, de los pueblos indígenas, cuya cosmovisión lleva a un vínculo importante de sus creencias con su territorio y, por tanto, la violación de los derechos ligados a éste con la violación del derecho a la LR. Se trata de algo muy específico del continente americano, y muy distinto del reconocimiento que las confesiones religiosas han experimentado en la jurisprudencia del TEDH, ligado al posicionamiento de su autonomía en el núcleo del derecho a la LR como consecuencia lógica de la neutralidad e imparcialidad del Estado.

La relación del derecho a la LR con la protección de los más vulnerables en el SIDH no la encontramos solo en ese trato específico a los pueblos indígenas. Posiblemente, el hecho de que parte de la actividad del SIDH se haya producido durante la pervivencia de sistemas dictatoriales ha generado también la relación entre actividades de oposición política y en defensa de los vulnerables con el derecho de LR de muchos de esos activistas.

\footnotetext{
${ }^{109}$ Corte IDH. El hábeas corpus bajo suspensión de garantías. OC-8/87 30 ene. 1987, pár. 42 y puntos resolutivos.

${ }^{110}$ Corte IDH. Garantías judiciales en estados de emergencia. OC-9/87 6 oct. 1987, párs. 38 y 39, y puntos resolutivos.

${ }^{111}$ Cervantes, op. cit., p. 132.
}

Araucaria. Revista Iberoamericana de Filosofia, Política, Humanidades y Relaciones Internacionales, año $23, \mathrm{n}^{\circ} 46$. Primer cuatrimestre de 2021. Pp. 591-612. ISSN 1575-6823 e-ISSN 2340-2199 https://dx.doi.org/10.12795/araucaria.2021.i46.29 
Mientras en Europa, el vínculo principal con lo político está en el constante recurso al MAN que prima la opción política-social de las mayorías sociales de los Estados europeos expresada, con frecuencia, jurídicamente. De forma que en ocasiones parece olvidarse el papel que ha de jugar el TEDH en la salvaguarda de los derechos de las personas, y especialmente las minorías, respecto al Estado y las mayorías. Son paradigmáticos los conflictos respecto a los símbolos religiosos, tan presentes en el escenario europeo y tan desconocidos para el SIDH, en los que el TEDH hace un uso especialmente generoso del MAN, el cual deja un amplio campo de acción a los Estados.

El SIDH no ha experimentado la evolución acontecida en la jurisprudencia del TEDH respecto a la objeción de conciencia y mantiene la posibilidad de su reconocimiento en la libertad regulatoria de los Estados. Pero ambos sistemas de protección coinciden, sobre todo tras la evolución experimentada por el TEDH, en una relación entre LR y libertad de expresión que permite afectar los sentimientos religiosos siempre que no se incurra en la apología del odio y la violencia.

Aunque en el artículo 12.3 de la CADH la presentación de los límites del derecho es más somera que en el 9.2 del CEDH, podría producirse una tendencia a la confluencia como sociedades democráticas; si bien, el SIDH se muestra más garantista. La referencia a la norma con rango de ley es formal y, por tanto, mucho más estricta, que la realizada por el TEDH. La CADH no permite la suspensión de este derecho en estados de emergencia, como sí lo autoriza el CEDH. Pero, sobre todo, la frecuencia del uso del MAN para primar expresiones estructurales de las mayorías religiosas de los Estados respecto a usos estrictos de pruebas de proporcionalidad por parte del TEDH, contrasta con el control de convencionalidad desarrollado por la Corte IDH que al obligar a todas las autoridades públicas de los Estados parte a seguir sus criterios, ha de fijar unos estándares claros e inequívocos a través de sus interpretaciones del contenido de los derechos.

En definitiva, la realidad misma de las sociedades americanas ha llevado al conocimiento de un derecho a la LR centrado en la protección de los más vulnerables (los pobres, los indígenas...). En Europa, la proliferación de conflictos parece expresar un proceso de acomodación de sociedades hasta hace relativamente poco muy uniformes en lo religioso y con confesiones religiosas muy cercanas a los poderes públicos, con nuevas realidades en las que prima la pluralidad de creencias y convicciones y sus correspondientes manifestaciones, que el Estado debería garantizar con neutralidad e imparcialidad. 


\section{Referencias bibliográficas:}

Avilés Hernández, Elena, "El discurso del odio como límite a la libertad de expresión: Análisis de la jurisprudencia del TEDH y su influencia en el Tribunal constitucional español”, [En Carmen Sánchez Hernández, María Fernanda Palam, Octavio García Pérez y Miguel Prata Roque, dirs., La influencia de la jurisprudencia del TEDH en el derecho interno, Valencia, Tirant lo Blanch, 2019, pp. 55-82].

Arlettaz, Fernando, "El individuo como titular de la libertad religiosa: aproximación a un problema de filosofía jurídica", [En $\mathrm{M}^{\mathrm{a}}$ Carmen Barranco Avilés, Óscar Celador Angón, Félix Vacas Fernández, coords., Perspectivas actuales de los sujetos de Derechos, Madrid, Dykinson y Universidad Carlos III de Madrid, 2012, pp. 303-315].

Arlettaz, Fernando, "La libertad religiosa en el Sistema Interamericano de derechos humanos", Revista Internacional de Derechos Humanos, 1, (2011), pp. 39-58.

Burgorgue-Larse, Laurence, "Le système interaméricain des droits de l'homme face aux religions", [En Robert Uerpmann-Wittzack, Evelyne Lagrange y Stefan Oeter, Religion and International Law. Living Together, LeidenBoston, Brill Nijhoff, 2018, pp. 157-174].

Cervantes G., Luis Francisco, "Los principios generales sobre la libertad religiosa en la jurisprudencia de los sistemas europeo, interamericano y costarricense de protección de los derechos humanos", Senderos: revista de ciencias religiosas y pastorales, 93 (2009), pp. 271-309.

Chueca Sancho, Ángel G., "El derecho humano a la libertad de religión y convicciones en una Europa intercultural", [En Teoría de la Justicia y Derechos Fundamentales. Estudios en Homenaje al profesor Gregorio Peces-Barba. Volumen III, Madrid, Dykinson, 2008, pp. 297-318].

Combalía, Zoila, "Los conflictos entre libertad de expresión y religión: tratamiento jurídico del discurso del odio", Anuario de Derecho Eclesiástico del Estado, XXXI (2015), pp. 355-379.

European Court of Human Rights. Research Division, Overview of the Court's case-law on freedom of religion, 2013. Disponible en: https://www.echr. coe.int/Documents/Research_report_religion_ENG.pdf

Fernández Arribas, Gloria, "La libertad religiosa en la Jurisprudencia el Tribunal Europeo de Derechos Humanos", [En Abraham Barrero Ortega y Manuel José Terol Becerra, coords.: La libertad religiosa en el Estado Social, Valencia, Tirant lo Blanch, 2009, pp. 443-462]. 
Fernández Parra, Sergio Alejandro, "El margen de apreciación nacional y el contenido de la libertad de pensamiento, conciencia y religión en el Convenio Europeo de Derechos Humanos", Eunomía. Revista en Cultura de la Legalidad, 7 (2019-2020), pp. 68-99.

Fix-Zamundio, Héctor, "La libertad religiosa en el Sistema Interamericano de protección de los derechos humanos", [En VV. AA.: La libertad religiosa. Memoria del IX Congreso internacional de Derecho canónico, México D. F., Instituto de Investigaciones Jurídicas de la Universidad Autónoma de México, 1996, pp. 499-510].

García Ureta, Agustín, "Libertad de pensamiento, de conciencia y de religión", [En Lasagabaster Herrarte, Iñaki: Convenio Europeo de Derechos Humanos. Comentario sistemático, Pamplona, Thomson Reuters, 2015, pp. 439-509].

Leigh, Ian, "New trends in Religious Liberty and the European Court of Human Rights", Ecclesiastical Law Society 12 (2020), pp. 266-279.

Martín Sánchez, Isidoro, "Las confesiones religiosas y su autonomía en el Tribunal Europeo de Derechos Humanos", Encuentros Multidisplinares, 26 (2014), pp. 1-9.

Martín-Retortillo Baquer, Lorenzo, "La libertad religiosa en la reciente jurisprudencia del Tribunal Europeo de Derechos Humanos", Anuario de Derecho Eclesiástico del Estado, XXVI (2010), pp. 287-332.

Palomino, Rafael, "Libertad religiosa y libertad de expresión”, Ius Canonicum, XLIX, 98 (2009), pp. 509-548.

Roca, María J., "Impacto de la jurisprudencia del TEDH y la Corte IDH sobre libertad religiosa", Revista Española de Derecho Constitucional, 110 (2017), pp. 253-281.

Romero Pérez, Xiomara Lorena, "La libertad religiosa en el Sistema Interamericano de Protección de los Derechos Humanos (Análisis Comparativo con el ordenamiento jurídico colombiano)", Revista Derecho del Estado, 29 (2012), pp. 215-232.

Scharffs, Brett G., "Symposium Introduction: The Freedom of Religion and Belief Jurisprudence of the European Court of Human Rights: Legal, Moral, Political and Religious Perspectives", Journal of Law and Religion, vol. 26, 1 (2010-2011), pp. 249-260.

Valero Estarellas, María José, “Autonomía institucional de las confesiones religiosas y derecho al respeto de la vida privada y familiar en Estrasburgo: la sentencia de la Gran Sala del TEDH Fernández Martínez c. España”, Revista General de Derecho Canónico y Derecho Eclesiástico del Estado, 36 (2014), pp. 1-21. 